\title{
Empirical Study on the Performance of Patent Strategy of China
}

\author{
Zhongguo Shi ${ }^{1,2}$, Jing $\mathrm{Li}^{2}$ \\ ${ }^{1}$ School of Management and Economics, University of Electronic Science and Technology, Chengdu, China; ${ }^{2}$ Institute of Electronic \\ Science and Technology, University of Electronic Science and Technology, Chengdu, China. \\ E-mail: shizg@uestc.edu.cn
}

Received August 13 ${ }^{\text {th }}, 2011$; revised October 11 ${ }^{\text {th }}, 2011$; accepted November $5^{\text {th }}, 2011$.

\begin{abstract}
Since the middle age of 1980s, China has made great performance at economic growth, and greatly improved its innovation level. As the representation index of innovation activity, patents number growth is also significant. This paper constructed quintic overdetermined equation of one variable to simulate the trend of patent number varying with the time from 1985 to 2010, made use of Matlab Software and took the solution of simulation model. By comparing the simulation curve and real data curve, good agreement is obtained. After F-test and comparison between the simulation data and real data of 2008, 2009, 2010 respectively, it is believed that the simulation model is reliable. Based on this model, scientific estimation about the variation of Chinese patents from 2011 to 2014 is presented.
\end{abstract}

Keywords: Patent, Simulation Model, Overdetermined Equation, Matlab

\section{Introduction}

Since 1980s, world economy structure is undergoing a new round of major adjustment. High technology industry rises quickly, which radiates and drives the development of the whole economy. National trades and investment activities are increasingly active, the competition between countries and enterprises is more intensive. This entire situation makes global economy, science and technology development pattern undergo a profound and significant change. The overall trend of world economy growth has had a profound and important influence on the international protection of intellectual property rights. Economic competition between countries has already been translated into competition of patents. For developed countries, patent strategy is one part of its global strategy to monopolize the global market. More and more countries and enterprises realize that intellectual property is the most important strategic resource for improving their core competition capabilities [1].

As a member of WTO, China has its crucial task to develop high-tech industries in order to meet the challenge of globalization. In Jun 5, 2008, the Sate Council promulgated "Outline of the national intellectual property strategy", decided to put intellectual property strategy into effect. Based on this situation, it is significant to find the rule of change and supply some advices for government decisions by studying on the trend of patents quantities in China from the middle age of 1980s till now.

\section{Data Source and Model Design}

From the website of State Intellectual Property Office (SIPO), we can find the whole sum of patents of China from 1985 to 2010, including invention patent, utility model patent and design patent, as shown in Table 1. In this paper, according to the patents number of each year, a simulation model of overdetermined equation group is constructed to describe the variation and developmental trends of patents from 1985 to 2007. To verify the fitting degree of the simulation model, patents data of 2008, 2009 and 2010 forecasted by the simulation model was compared with the real data published at the website of SIPO. Additionally, statistics test methods such as $F$ test are used to verify the fitting degree. The whole solving process is underdone by Matlab software.

According to the data shown in Table 1, a trend curve of the number of patents (observed value) varying with the time (year) is drawn. Assume that there has one parallel curve intersect with the trend curve, $k$ points are obtained. Regarding time (year) $X_{t}$ as independent variable, number of patents $Y_{i}$ as dependent variable, $k$ overdetermined equation of one variable is constructed [2,3], as shown in Equation (1). 
Table 1. Data of patens of China from 1985 to 2007.

\begin{tabular}{|c|c|c|c|c|c|c|c|}
\hline \multirow{2}{*}{ Year } & \multirow{2}{*}{$\begin{array}{c}\text { Whole Sum of Patents } \\
\text { Number }\end{array}$} & \multicolumn{2}{|c|}{ Invention Patens } & \multicolumn{2}{|c|}{ Utility Model Patents } & \multicolumn{2}{|c|}{ Design Patents } \\
\hline & & Number & Percent (\%) & Number & Percent (\%) & Number & Percent (\%) \\
\hline 2007 & 351782 & 67948 & 19.32 & 150036 & 42.65 & 133798 & 38.03 \\
\hline 2006 & 268002 & 57786 & 21.56 & 107655 & 40.17 & 102561 & 38.27 \\
\hline 2005 & 214003 & 53305 & 24.91 & 79349 & 37.08 & 81349 & 38.01 \\
\hline 2004 & 190238 & 49360 & 25.95 & 70623 & 37.12 & 70255 & 36.93 \\
\hline 2003 & 182226 & 37154 & 20.39 & 68906 & 37.81 & 76166 & 41.80 \\
\hline 2002 & 132401 & 21476 & 16.22 & 57483 & 43.42 & 53442 & 40.36 \\
\hline 2001 & 114252 & 16297 & 14.26 & 54359 & 47.58 & 43596 & 38.16 \\
\hline 2000 & 105345 & 12683 & 12.04 & 54743 & 51.97 & 37919 & 36.00 \\
\hline 1999 & 100156 & 7637 & 7.63 & 56368 & 56.28 & 36151 & 36.09 \\
\hline 1998 & 67889 & 4733 & 6.97 & 33902 & 49.94 & 29254 & 43.09 \\
\hline 1997 & 50996 & 3494 & 6.85 & 27342 & 53.62 & 20160 & 39.53 \\
\hline 1996 & 43781 & 2977 & 6.80 & 27171 & 62.06 & 13633 & 31.14 \\
\hline 1995 & 45064 & 3393 & 7.53 & 30471 & 67.62 & 11200 & 24.85 \\
\hline 1994 & 43297 & 3883 & 8.97 & 32819 & 75.80 & 6595 & 15.23 \\
\hline 1993 & 62127 & 6556 & 10.55 & 46639 & 75.07 & 8932 & 14.38 \\
\hline 1992 & 31475 & 3966 & 12.60 & 24060 & 76.44 & 3449 & 10.96 \\
\hline 1991 & 24616 & 4122 & 16.75 & 17327 & 70.39 & 3167 & 12.87 \\
\hline 1990 & 22588 & 3838 & 16.99 & 16952 & 75.05 & 1798 & 7.96 \\
\hline 1989 & 17129 & 2303 & 13.45 & 13508 & 78.86 & 1318 & 7.69 \\
\hline 1988 & 11947 & 1025 & 8.58 & 10191 & 85.30 & 731 & 6.12 \\
\hline 1987 & 6811 & 422 & 6.20 & 5768 & 84.69 & 621 & 9.12 \\
\hline 1986 & 3024 & 56 & 1.85 & 2530 & 83.66 & 438 & 14.48 \\
\hline 1985 & 138 & 40 & 28.99 & 60 & 43.48 & 38 & 27.54 \\
\hline
\end{tabular}

a. Data Source: website of SIPO of China http://www.sipo.gov.cn/tjxx/.

$$
y_{t}=a_{1} x_{t}^{k}+a_{2} x_{t}^{k-1}+\cdots+a_{k-1} x_{t}^{2}+a_{k} x_{t}+a_{k+1}
$$

In which, $k \geq 1, a_{1}, a_{2}, \ldots, a_{k+1}$ are constants.

In matrix forms, Equation (1) can be written as follows,

$$
\mathbf{Y}_{t \times 1}=\mathbf{X}_{t \times(k+1)} \mathbf{A}_{(k+1) \times 1}
$$

In which,

$$
\begin{gathered}
\mathbf{Y}_{t \times 1}=\left[y_{1}, y_{2}, \cdots, y_{t}\right]^{\mathrm{T}}, \mathbf{A}_{(k+1) \times 1}=\left[a_{1}, a_{2}, \cdots, a_{k+1}\right]^{\mathrm{T}}, \\
\mathbf{X}_{t \times(k+1)}=\left[\begin{array}{ccccc}
x_{1}^{k} & x_{1}^{k-1} & \cdots & x_{1}^{1} & 1 \\
x_{2}^{k} & x_{2}^{k-1} & \cdots & x_{2}^{1} & 1 \\
\vdots & & \ddots & \vdots & \vdots \\
x_{t}^{k} & x_{t}^{k-1} & \cdots & x_{t}^{1} & 1
\end{array}\right]
\end{gathered}
$$

\section{Account Case}

Take the year number shown in Table $\mathbf{1}$ into $\mathbf{Y}_{t \times 1}$ of equation (2), the data of patents into equation (3), $K$ overdetermined equation of one variable about parameter
$\mathbf{A}_{(k+1) \times 1}$ is gotten. Under this condition, parameter $\mathbf{A}_{(k+1) \times 1}$ of Equation (2) has a least square solution $\mathbf{A}_{(k+1) \times 1}$, thus the equation $\left\|\mathbf{X}_{t \times(k+1)} \hat{\mathbf{A}}_{(k+1) \times 1}-\mathbf{Y}_{t \times 1}\right\|_{2}$ has a minimum sotion [4] which could be expressed as:

$$
\left\|\mathbf{X}_{t \times(k+1)} \hat{\mathbf{A}}_{(k+1) \times 1}-\mathbf{Y}_{t \times 1}\right\|_{2}=\min \left\|\mathbf{X}_{t \times(k+1)} \mathbf{A}_{(k+1) \times 1}-\mathbf{Y}_{t \times 1}\right\|_{2}
$$

\subsection{About the Total Amount of Patents}

Make use of Equation (1), and appoint $k=5$, a quintic polynomial is utilized to process fitting. The total amount of patents is $Y_{0}$, time number is $x_{t}$, and then the total amount of patents varying with the time can be expressed as:

$$
Y_{0}=a_{1} x_{t}^{5}+a_{2} x_{t}^{4}+a_{3} x_{t}^{3}+a_{4} x_{t}^{2}+a_{5} x_{t}+a_{6}
$$

Take the data "whole Sum of Patents" shown in Table 1 into (5), and dissolve the overdetermined equation group 
constructed by (5), solutions of parameters are: $a_{1}=0.27$, $a_{2}=-12.42, a_{3}=239.85, a_{4}=-2068.49, a_{5}=2010.36$ and $a_{6}=-12982.78$. The comparison figure between simulation curve and real curve is shown in Figure 1.

\subsection{About the Invention Patents}

The same as section 3.1, appoint the number of invention patents is $Y_{1}$ and time (year) is $x_{t}$, number of patents varying with time can be expressed as:

$$
Y_{1}=a_{1} x_{t}^{5}+a_{2} x_{t}^{4}+a_{3} x_{t}^{3}+a_{4} x_{t}^{2}+a_{5} x_{t}+a_{6}
$$

Take the data "invention patents" shown in Table 1 and dissolve the overdetermined equation group constructed by (6), the solution of parameters can be obtained as: $a_{1}$ $=-0.322, a_{2}=18.33, a_{3}=-351.53, a_{4}=2761.10, a_{5}=$ -7793.88, $a_{6}=6216.43$. The comparison figure between simulation curve and real curve is shown in Figure 2.

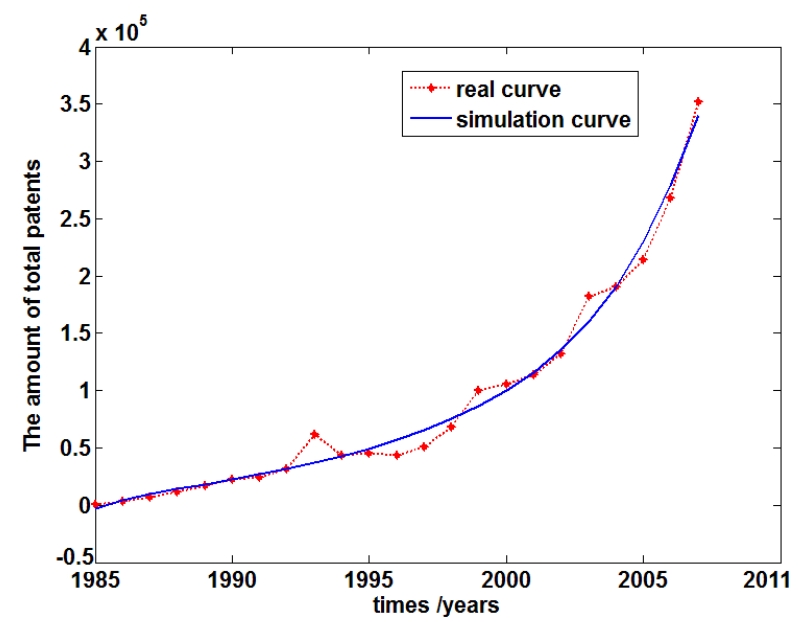

Figure 1. The comparison between simulation curve and real curve of whole sum of patents.

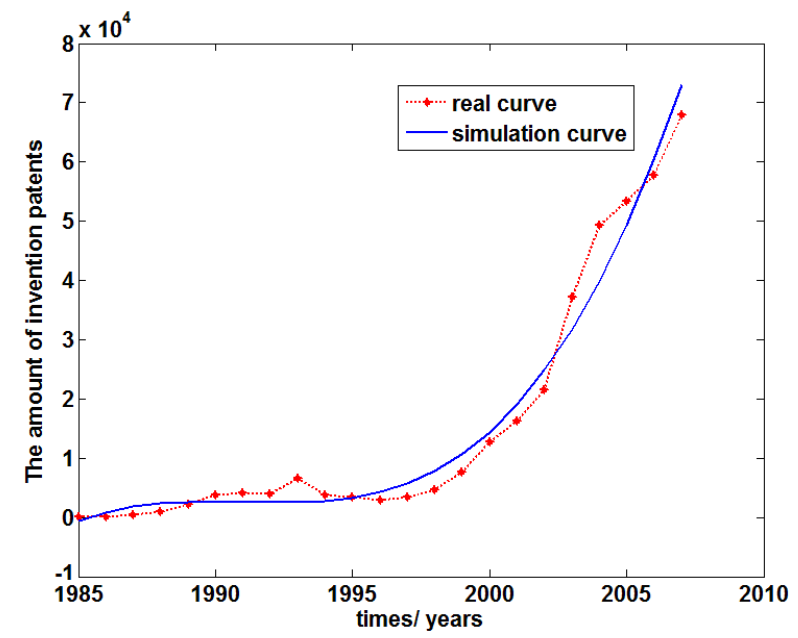

Figure 2. The comparison between simulation curve and real curve of invention patents.

\subsection{About the Utilize Model Patents}

The same as section 3.1, appoint the number of invention patents is $Y_{2}$ and the time (year) is $x_{t}$, the number of patents varying with time can be expressed as:

$$
Y_{2}=a_{1} x_{t}^{5}+a_{2} x_{t}^{4}+a_{3} x_{t}^{3}+a_{4} x_{t}^{2}+a_{5} x_{t}+a_{6}
$$

Take the data "invention patents" shown in Table 1 and dissolve the overdetermined equation group constructed by (6), the solutions of parameters are obtained as: $a_{1}=$ $-0.31, a_{2}=-15.20, a_{3}=272.86, a_{4}=-2213.33, a_{5}=$ 11154.51, $a_{6}=-11638.51$. The simulation curve and the real curve are shown in Figure 3.

\subsection{About the Design Patents}

The same as section 3.1, appoint the number of invention patents is $Y_{3}$ and the time (year) is $x_{t}$, the number of patents varying with time can be expressed as:

$$
Y_{3}=a_{1} x_{t}^{5}+a_{2} x_{t}^{4}+a_{3} x_{t}^{3}+a_{4} x_{t}^{2}+a_{5} x_{t}+a_{6}
$$

Take the data "design patents" shown in Table 1 and dissolve the overdetermined equation group (8), the solution of parameters are achieved as: $a_{1}=0.28, a_{2}=-15.82$, $a_{3}=325.24, a_{4}=-2691.60, a_{5}=9023.94, a_{6}=-8186.80$. The comparison between simulation curve and real curve is shown in Figure 4.

\subsection{Forecast data}

From the above description, it is believed that the fitting degree between the simulated and real curve is good. The simulation model can be used to forecast the data of coming years. The forecast patents data of 2011, 2012, 2013 and 2014 are shown in Table 2.

From Table 2, it can be seen that the growth rate of patents keeps about $30 \%$ in the coming years, and the growth rate of invention patents is slower than those of other two kinds of patents.

\section{Test Method and Result}

\subsection{F-Test}

In order to test the reliability of the simulation model, $F$ test method is used as below.

Total sum of squares of deviations:

$$
Q_{T}=\sum_{i=1}^{n}\left(y_{i}-\bar{y}\right)^{2}
$$

Equation (9) described the total dispersion degree of the observe value $y_{1}, y_{2}, \cdots y_{n}$ for dependable variable $Y$. Decompounds $Q_{T}$ to two parts, that is, square sum of residuals $\left(Q_{E}\right)$ and sum of squares of deviations in regression $\left(Q_{R}\right)$

$$
Q_{T}=Q_{E}+Q_{R}
$$




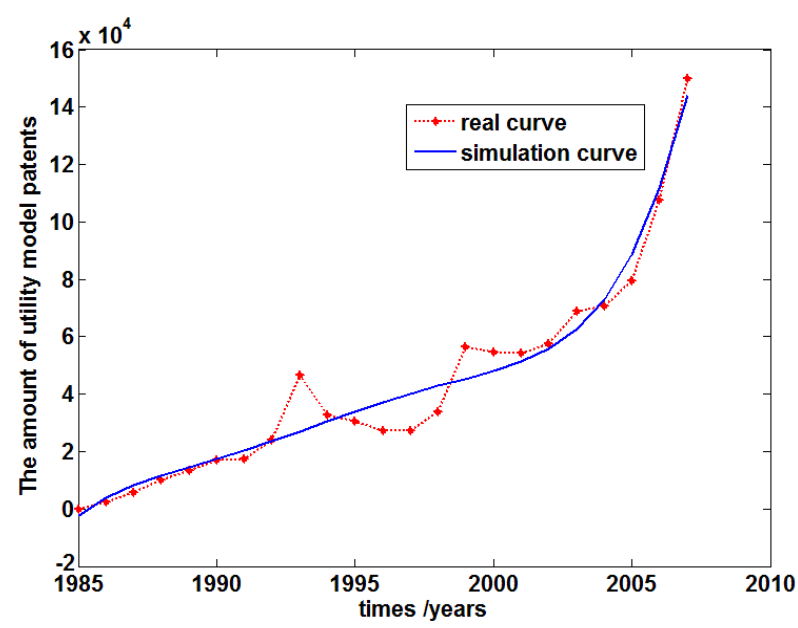

Figure 3. The comparison between simulation curve and real curve of utilize model patents.

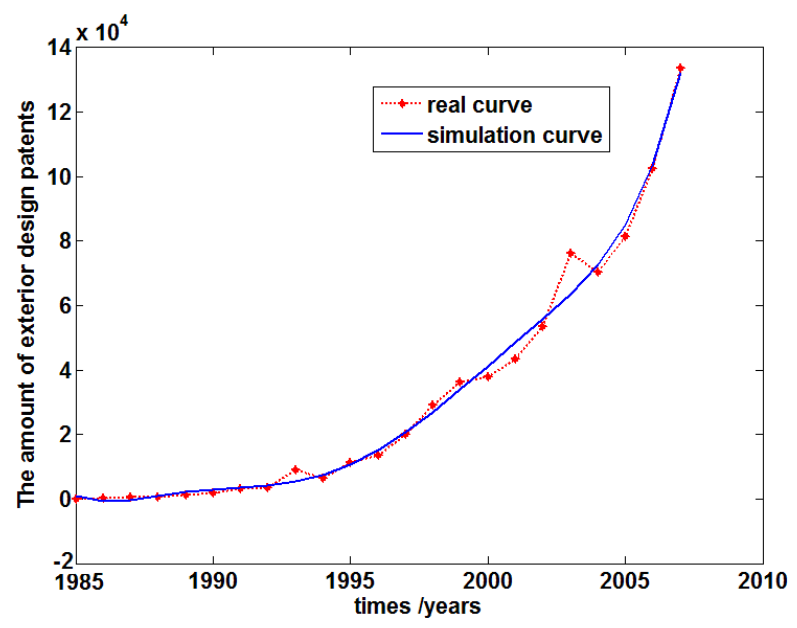

Figure 4. The comparison between simulation curve and real curve of design patents.

Table 2. The forecast data of the coming years.

\begin{tabular}{ccccccccc}
\hline \multirow{2}{*}{ Year } & \multicolumn{2}{c}{ Whole Sum of Patents } & \multicolumn{2}{c}{ Invention Patens } & \multicolumn{2}{c}{ Utility Model Patents } & \multicolumn{2}{c}{ Design Patents } \\
\cline { 2 - 8 } & Number & Growth Rate & Number & Growth Rate & Number & Growth Rate & Number & Growth Rate \\
\hline 2011 & 1059688 & 30.05 & 165191 & 22.26 & 437133 & 26.89 & 457454 & 36.45 \\
2012 & 1407002 & 32.27 & 194591 & 17.79 & 585540 & 33.94 & 627082 & 37.08 \\
2013 & 1853821 & 31.75 & 227576 & 16.95 & 776802 & 32.66 & 849842 & 35.52 \\
2014 & 2420316 & 30.56 & 264400 & 16.18 & 1019460 & 31.23 & 1137133 & 33.81 \\
\hline
\end{tabular}

Table 3. Compare between forecast data and real data of 2008-2010.

\begin{tabular}{cccccc}
\hline & Year & Whole Sum of Patents & Invention Patents & Utilize Model Patents & Design Patents \\
\hline \multirow{3}{*}{2010} & Real Data & 814825 & 135110 & 344472 & 335243 \\
& Forecast data & 632683 & 121381 & 331258 & 353126 \\
& Error & $-22.35 \%$ & $-10.16 \%$ & $-3.83 \%$ & $5.33 \%$ \\
\multirow{2}{*}{2009} & Real Data & 581992 & 128489 & 203802 & 249701 \\
& Forecast data & 513895 & 103381 & 250291 & 248039 \\
& Error & $-11.70 \%$ & $-19.54 \%$ & $22.81 \%$ & $-0.66 \%$ \\
& Real Data & 411982 & 93706 & 176675 & 141601 \\
& Forecast data & 417802 & 87284 & 189210 & $7.09 \%$ \\
\hline
\end{tabular}

In which

$$
Q_{E}=\sum_{i=1}^{n}\left(y_{i}-\hat{y}_{i}\right)^{2} ; \quad Q_{R}=\sum_{i=1}^{n}\left(\hat{y}_{i}-\bar{y}\right)^{2}
$$

test statistics:

$$
F=\frac{Q_{R} / k}{Q_{E} /(n-k-1)} \sim F(k, n-k-1)
$$

As for the given significance $\alpha$, test whether $F$ is bigger than $F_{\alpha}$.

- $\quad$ To test Equation (5), $n=23, k=6$

We can get $Q_{T}=1.9211 \mathrm{e}+011 ; Q_{R}=1.8976 \mathrm{e}+011$;
$Q_{E}=2.3542 \mathrm{e}+009 . F=214.9479>F_{0.01}(6,16)=4.20$.

- $\quad$ To test Equation (6)

We can get $Q_{T}=9.9524 \mathrm{e}+009 ; Q_{R}=9.8738 \mathrm{e}+009$; $Q_{E}=7.8567 \mathrm{e}+007 . F=335.1285>F_{0.01}(6,16)=4.20$.

- To test Equation (7)

We can get $Q_{T}=2.8411 \mathrm{e}+010 ; Q_{R}=2.7276 \mathrm{e}+010$; $Q_{E}=1.1346 \mathrm{e}+009 . F=64.1051>F_{0.01}(6,16)=4.20$.

- $\quad$ To test Equation (8)

We can get $Q_{T}=3.1413 \mathrm{e}+010 ; Q_{R}=3.1093 \mathrm{e}+010$; $Q_{E}=3.2013 \mathrm{e}+008 . F=259.0053>F_{0.01}(6,16)=4.20$.

From the test result, we can conclude that Equation (5), (6), (7) and (8) all reach the significance level. 


\subsection{Observe Data Test}

We can also use the simulation model to forecast the patents number of 2008, 2009, and 2010, thus further test the significance of the model by comparing the simulation data with the real data published at the website of SIPO. The results are shown in Table 3.

From the above table, it can be concluded that the maximum error between the forecast data and real data is about $20 \%$, the minimum error is less than $1 \%$. The errors are tolerable, thus the simulation model is effective.

\section{Conclusions}

Firstly, the trend of patents number varying with time from 1985 to 2010 in China can be explained by quintic overdetermined equation of one variable. Three kinds of patens, that is, invention patent, utilize model patent and design patent, are all showing the same trend. The fitting degree is well. Under the F-test and observe data test, the significance level of the simulation model is also good. Secondly, from the simulation model, we can get the forecast data about the several coming years. It is helpful for decision support. Thirdly, the distinction between three kinds of patents is that the growth rate is different, invention patent is slowest, and design patent is fastest. This phenomenon should be taken into consideration. And finally, the curve is steep, especially after 2000, it shows that self-innovation strategy of China has taken great effect in the decades.

\section{REFERENCES}

[1] S.-J. Zhang, "Literature Review of Patent Strategy Study in China,” Management of Science and Technology, Vol. 2, 2011, pp. 174-175.

[2] Z.-Q. GE and J. Yang, "The Experimental Study on the Patent Application of Universities and Colleges in China,” Journal of UESTC of China, Vol. 35, No. 2, 2006, pp. 285-286.

[3] Z.-Q. GE, B. Zhang and T. Jin, “An Empirical Study on the Relationship between Input of R \& D and Patent in China," Proceedings of 2004 International Conference on Management Science and Engineering, Orient Academic Forum Beijing-Sidney, September 15-25, 2004, pp. 118120.

[4] B.-J. Li and H.-Y. Liu, "A Solving Method of Overdetermined Systems," Journal of Shenyang University of Technology, Vol. 24, No.1, 2002, pp. 76-77. 